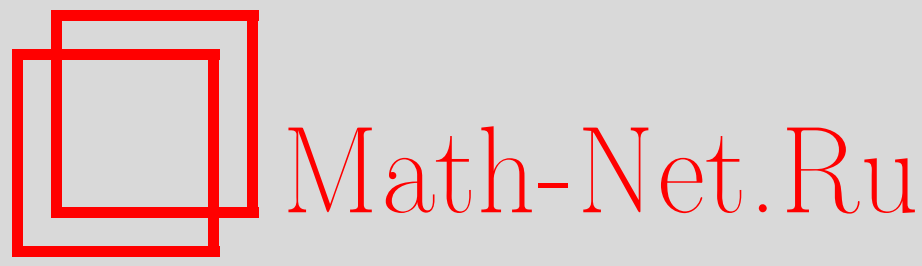

А. Г. Сергеев, Твисторное квантование пространств петель компактных групп Ли, ТМФ, 2008, том 157, номер 3, 450-467

DOI: https://doi.org/10.4213/tmf6292

Использование Общероссийского математического портала Math-Net.Ru подразумевает, что вы прочитали и согласны с пользовательским соглашением http://www . mathnet.ru/rus/agreement

Параметры загрузки:

IP : 54.224 .60 .19

26 апреля 2023 г., 16:11:50

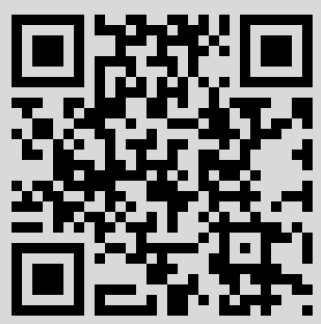




\section{ТВИСТОРНОЕ КВАНТОВАНИЕ ПРОСТРАНСТВ ПЕТЕЛЬ КОМПАКТНЫХ ГРУПП ЛИ}

Рассмотрена задача твисторного квантования для пространства петель $\Omega_{T} G$ компактной группы Ли $G$. Показано, что эта задача разрешима в критической размерности.

Ключевые слова: твисторное квантование, пространство петель, фоковское расслоение, аффинные алгебры, действие Гудмана-Уолаха.

Посвящается Василию Сергеевичу Владимирову в связи с 85-летием

Пространство петель $\Omega_{T} G=L G / T$ является фактором группы петель $L G:=$ $C^{\infty}\left(S^{1}, G\right)$ компактной группы Ли $G$ по модулю постоянных отображений $S^{1} \rightarrow T$ в максимальный тор $T$ группы $G$. Это пространство, которое можно рассматривать как естественный бесконечномерный аналог многообразия полных флагов $F=G / T$, играет в теории представлений групп петель такую же роль, какую многообразие полных флагов играет в теории представлений компактных групп Ли. В частности, неприводимые представления группы петель $L G$ реализуются с помощью конструкции Бореля-Вейля в пространствах голоморфных сечений комплексных линейных расслоений над $\Omega_{T} G$. Указанная конструкция лежит в основе твисторного квантования пространства $\Omega_{T} G$. Кроме того, пространство $\Omega_{T} G$ тесно связано с теорией струн, будучи фазовым многообразием этой теории, что делает геометрическое квантование указанного пространства важной и интересной задачей. Твисторное квантование пространства $\Omega_{T} G$, составляющее основной предмет настоящей статьи, можно рассматривать как промежуточный этап в построении геометрического квантования $\Omega_{T} G$.

Напомним, что твисторное квантование фазового многообразия $M$ означает построение гильбертова расслоения над пространством допустимых комплексных структур на $M$ вместе с плоской эрмитовой связностью на нем. В нашем случае роль фазового пространства играет пространство петель $\Omega_{T} G$. Мы наделяем это бесконечномерное многообразие алгеброй наблюдаемых $\mathcal{A}$, задаваемой полупрямым

${ }^{*}$ Математический институт им. В. А. Стеклова РАН, Москва, Россия. E-mail: sergeev@mi.ras.ru 
произведением $L \mathfrak{g} \rtimes \operatorname{Vect}\left(S^{1}\right)$ алгебры петель $L \mathfrak{g}$, где $\mathfrak{g}$ - алгебра Ли группы $G$, и алгебры $\operatorname{Vect}\left(S^{1}\right)$ касательных векторных полей на окружности. Допустимые комплексные структуры на $\Omega_{T} G$ получаются из стандартной комплексной структуры действием группы диффеоморфизмов $\operatorname{Diff}_{+}\left(S^{1}\right)$, задаваемым репараметризацией петель. Пространство допустимых комплексных структур отождествляется с фактором $\mathcal{S}:=\operatorname{Diff}_{+}\left(S^{1}\right) / \operatorname{Möb}\left(S^{1}\right)$ этой группы по модулю автоморфизмов Мёбиуса единичного круга, суженных на $S^{1}$. Гильбертово расслоение квантования $\mathcal{H}$ на $\mathcal{S}$ совпадает с фоковским расслоением полуформ над $\mathcal{S}$. Его можно снабдить естественным проективным действием группы диффеоморфизмов $\operatorname{Diff}_{+}\left(S^{1}\right)$, построенным в работе Гудмана-Уолаха [1]. Это действие порождает эрмитову связность на $\mathcal{H}$, которая является плоской в критической размерности.

Коротко о содержании статьи. В разделе 1 вводится пространство петель $\Omega_{T} G$ и определяются его комплексная и симплектическая структуры. Затем рассматривается действие группы диффеоморфизмов $\operatorname{Diff}_{+}\left(S^{1}\right)$ на $\Omega_{T} G$ и изучается пространство допустимых комплексных структур на $\Omega_{T} G$. В разделе 2 мы напоминаем хорошо известные факты о квантовании Дирака и предквантовании Костанта-Сурьо (KS-предквантование). Раздел 3 содержит базисные сведения о структуре аффинных алгебр и их представлениях старшего веса. Раздел 4 посвящен конструкции Бореля-Вейля для групп петель [2]. В разделе 5 представлена конструкция Гудмана-Уолаха, с помощью которой строится проективно плоская унитарная связность на фоковском расслоении. Раздел завершается построением расслоения квантования, отождествляемого с фоковским расслоением полуформ на $\mathcal{S}$. Это расслоение наделяется эрмитовой связностью, которая является плоской в критической размерности.

\section{1. КЭЛЕРОВА ГЕОМЕТРИЯ ПРОСТРАНСТВА $\Omega_{T} G$}

1.1. Пространство $\Omega_{T} G$. Пусть $G$ - компактная связная группа Ли, $T$ - максимальный тор в $G$. Рассмотрим пространство петель $\Omega_{T} G$, являющееся однородным пространством группы петель $L G=C^{\infty}\left(S^{1}, G\right)$ вида

$$
\Omega_{T} G=L G / T \text {. }
$$

Группа петель $L G=C^{\infty}\left(S^{1}, G\right)$ есть группа гладких отображений окружности $S^{1}=$ $\{|z|=1\} \subset \mathbb{C}$ в группу $G$, а $T$ отождествляется с группой постоянных отображений $S^{1} \rightarrow g_{0} \in T$. Пространство петель $\Omega_{T} G$ есть однородное пространство группы Ли-Фреше $L G$ с естественным действием $L G$ левыми сдвигами.

С теоретико-групповой точки зрения группа петель $L G$ является полупрямым произведением группы $G$ и подгруппы $L_{1} G$ отображений из $L G$, переводящих $1 \in S^{1}$ в единицу $e \in G$. Как многообразие Фреше группа петель $L G$ диффеоморфна прямому произведению $G \times \Omega G$, где

$$
\Omega G=L G / G
$$

Заметим, что $\Omega G$ можно отождествить (как однородное пространство) с подгруппой $L_{1} G$, сопоставляя классу $[\gamma]$ петли $\gamma \in L G$ отображение $\gamma(1)^{-1} \gamma \in L_{1} G$. При 
таком отождествлении пространство $\Omega G$ реализуется в виде замкнутого подмногообразия (коразмерности 1 ) в многообразии Фреше $L G$ и поэтому является многообразием Фреше. Следовательно, многообразием Фреше является и пространство петель $\Omega_{T} G$, диффеоморфное

$$
\Omega_{T} G=L G / T=G / T \times \Omega G .
$$

Пространство $\Omega_{T} G$ обладает естественной симплектической структурой, которую можно ввести с помощью диффеоморфизма (2). Инвариантную (относительно левых сдвигов группы $L G$ ) симплектическую структуру на $\Omega G$ можно определить с помощью инвариантного (относительно присоединенного действия) скалярного произведения $\langle\cdot, \cdot\rangle$ на алгебре Ли $\mathfrak{g}$ группы $G$. Инвариантная симплектическая структура на $\Omega G$ полностью определяется ее сужением на касательное пространство $T_{o}(\Omega G)$ в начале $о$. Это касательное пространство совпадает с

$$
T_{o}(\Omega G)=\Omega \mathfrak{g}:=L \mathfrak{g} / \mathfrak{g},
$$

где $L \mathfrak{g}:=C^{\infty}\left(S^{1}, \mathfrak{g}\right)$ - алгебра петель со скобкой Ли, определяемой поточечно. Элементы $\xi \in \Omega \mathfrak{g}$ можно задавать их разложениями Фурье

$$
\xi\left(e^{i \theta}\right)=\sum_{k \neq 0} \xi_{k} e^{i k \theta},
$$

где $\xi_{k} \in \mathfrak{g}^{\mathbb{C}}, \xi_{-k}=\bar{\xi}_{k}$.

Рассмотрим 2-форму $\omega_{0}$ на алгебре петель $L \mathfrak{g}$, задаваемую формулой

$$
\omega_{0}(\xi, \eta)=\frac{1}{2 \pi} \int_{0}^{2 \pi}\left\langle\xi\left(e^{i \theta}\right), \frac{d \eta\left(e^{i \theta}\right)}{d \theta}\right\rangle d \theta
$$

на векторах $\xi=\xi\left(e^{i \theta}\right), \eta=\eta\left(e^{i \theta}\right)$ из алгебры петель $L \mathfrak{g}=C^{\infty}\left(S^{1}, \mathfrak{g}\right)$. Это кососимметричная билинейная замкнутая форма на $L \mathfrak{g}$, которая обращается в нуль, если хотя бы одно из отображений $\xi, \eta$ постоянно. Опуская форму $\omega_{0}$ на $\Omega \mathfrak{g}$, получим невырожденную кососимметричную билинейную замкнутую 2-форму $\omega$ на $\Omega \mathfrak{g}$, инвариантную относительно присоединенного действия группы $G$ на $\Omega \mathfrak{g}$. Эта форма продолжается (с помощью левых сдвигов) до $L G$-инвариантной симплектической формы $\omega$ на $\Omega G$.

Кроме того, мы можем использовать скалярное произведение $\langle\cdot, \cdot\rangle$ на $\mathfrak{g}$, чтобы отождествить однородное пространство $G / H$ с орбитой коприсоединенного представления группы $G$ в пространстве $\mathfrak{g}^{*}$, двойственном к алгебре Ли $\mathfrak{g}$. Такие орбиты обладают канонической симплектической структурой, задаваемой формой Кириллова [3]. Симплектические структуры на $\Omega G$ и $G / H$ порождают с помощью диффеоморфизма (2) симплектическую структуру на пространстве петель $\Omega_{T} G$.

Пространство $\Omega_{T} G$ можно также наделить инвариантной комплексной структурой $J$, которая индуцируется “комплексным" представлением $\Omega_{T} G$ в виде однородного пространства комплексифицированной группы петель $L G^{\mathbb{C}}$. Это представление имеет вид

$$
\Omega_{T} G=L G^{\mathbb{C}} / B_{+} G^{\mathbb{C}}
$$


где $B_{+} G^{\mathbb{C}}-$ подгруппа $L_{+} G^{\mathbb{C}}=\operatorname{Hol}\left(\Delta, G^{\mathbb{C}}\right)$, состоящая из отображений $\gamma \in$ $C^{\infty}\left(S^{1}, G^{\mathbb{C}}\right)$, продолжающихся до голоморфных и гладких вплоть до $S^{1}$ отображений $\gamma: \Delta \rightarrow G^{\mathbb{C}}$ единичного круга $\Delta$, которые удовлетворяют дополнительному условию: $\gamma(0) \in B_{+}$, где $B_{+}-$стандартная подгруппа Бореля в $G$. Доказательство этого утверждения можно найти в работе [2]. Кроме того, можно ввести комплексную структуру на $\Omega_{T} G$, пользуясь диффеоморфизмом (2). Искомая комплексная структура будет порождаться инвариантными комплексными структурами на $\Omega G$ и однородном пространстве $G / H$, рассматриваемом как многообразие флагов [4].

Введенная комплексная структура $J$ на $\Omega_{T} G$ совместима с симплектической формой $\omega$ и тем самым определяет на $\Omega_{T} G$ структуру кэлерова многообразия Фреше. Главной целью статьи является твисторное квантование этого многообразия.

1.2. Действие группы диффеоморфизмов на $\Omega_{T} G$. Группа $\operatorname{Diff}_{+}\left(S^{1}\right)$ диффеоморфизмов $S^{1}$, сохраняющих ориентацию, действует на группе петель $L G$ заменой переменной

$$
T_{f}(\gamma):=\gamma \circ f-\frac{1}{2 \pi} \int_{0}^{2 \pi} \gamma(f(\theta)) d \theta
$$

при $\gamma \in \Omega G, f \in \operatorname{Diff}_{+}\left(S^{1}\right)$. Это действие порождает симплектическое действие группы $\operatorname{Diff}_{+}\left(S^{1}\right)$ на $\Omega G$. Мы продолжаем его тривиальным образом на второй множитель $G / T$ в произведении $\Omega_{T} G=\Omega G \times G / T$.

Диффеоморфизм $f \in \operatorname{Diff}_{+}\left(S^{1}\right)$ преобразует комплексную структуру $J$ в комплексную структуру

$$
J_{f}:=f_{*}^{-1} \circ J \circ f_{*},
$$

где $f_{*}$ - отображение, касательное к $f$. Эта новая комплексная структура $J_{f}$ эквивалентна исходной комплексной структуре $J$ тогда и только тогда, когда $f \in \operatorname{Möb}\left(S^{1}\right)$. Здесь $\operatorname{Möb}\left(S^{1}\right)$ есть группа дробно-линейных автоморфизмов единичного круга, суженных на $S^{1}$.

Будем называть комплексные структуры $J_{f}=f_{*}^{-1} \circ J \circ f_{*}$ на $\Omega G$, полученные из $J$ действием группы диффеоморфизмов $\operatorname{Diff}_{+}\left(S^{1}\right)$, допустимыми. Пространство допустимых комплексных структур на $\Omega_{T} G$ отождествляется с многообразием $\mathcal{S}:=$ $\operatorname{Diff}_{+}\left(S^{1}\right) / \operatorname{Möb}\left(S^{1}\right)$.

Напомним, что комплексная структура $J$ на $\Omega_{T} G$ инвариантна относительно левых $L G$-сдвигов на $\Omega_{T} G$ и совместима с симплектической структурой $\omega$. С учетом инвариантности $\omega$ относительно действия группы Diff $+\left(S^{1}\right)$ комплексные структуры $J_{f}$ также инвариантны относительно левых $L G$-сдвигов и совместимы с $\omega$.

1.3. Пространство допустимых комплексных структур. Пространство допустимых комплексных структур

$$
\mathcal{S}:=\operatorname{Diff}_{+}\left(S^{1}\right) / \operatorname{Möb}\left(S^{1}\right)
$$

является кэлеровым многообразием Фреше. Для того чтобы показать это, введем комплексную структуру $I$ на $\mathcal{S}$, инвариантную относительно действия группы $\operatorname{Diff}_{+}\left(S^{1}\right)$ левыми сдвигами. Ввиду инвариантности достаточно определить ее в начале $o \in \mathcal{S}$. 
Касательное пространство $T_{o} \mathcal{S}$ отождествляется с факторпространством алгебры Ли $\operatorname{Vect}\left(S^{1}\right)$ касательных векторных полей на $S^{1}$ по модулю подалгебры $\operatorname{sl}(2, \mathbb{R})$. $\mathrm{B}$ терминах разложений Фурье векторные поля $v=v(\theta) d / d \theta \in T_{o} \mathcal{S}$ задаются рядами вида

$$
v(\theta)=\sum_{n \neq-1,0,1} v_{n} e^{i n \theta}, \quad v_{n} \in \mathbb{C},
$$

удовлетворяющими условию $v_{-n}=\bar{v}_{n}$. В этих терминах сужение $\operatorname{Diff}_{+}\left(S^{1}\right)$-инвариантной комплексной структуры $I$ на $T_{o} \mathcal{S}$ определяется формулой

$$
I v(\theta)=-i \sum_{n>1} v_{n} e^{i n \theta}+i \sum_{n<-1} v_{n} e^{i n \theta}
$$

для $v=v(\theta) d / d \theta \in T_{o} \mathcal{S}$. Эта комплексная структура совместима с симплектической структурой $w$ на $\mathcal{S}$, которая определяется в терминах разложений Фурье следующим образом.

На касательных векторах $u, v \in T_{o} \mathcal{S}$ с разложениями Фурье

$$
u=\sum_{n \neq-1,0,1} u_{n} e_{n}, \quad v=\sum_{n \neq-1,0,1} v_{n} e_{n}
$$

значение $w$ равно

$$
w(u, v)=2 \operatorname{Im} \sum_{n=2}^{\infty} u_{n} \bar{v}_{n}\left(n^{3}-n\right) .
$$

Симплектическая форма $w$ вместе с комплексной структурой $I$ определяют кэлерову метрику $g$ на $\mathcal{S}$. В терминах разложений Фурье указанная метрика задается формулой

$$
g(u, v)=2 \operatorname{Re} \sum_{n=2}^{\infty} u_{n} \bar{v}_{n}\left(n^{3}-n\right)
$$

на касательных векторах $u, v \in T_{o} \mathcal{S}$ с разложениями Фурье (5).

\section{2. КВАНТОВАНИЕ ДИРАКА}

2.1. Квантование классических систем. Напомним, что классическая (или механическая) система задается парой $(M, \mathcal{A})$, где $M$ - фазовое пространство системы, $\mathcal{A}$ - ее алгебра наблюдаемых (или гамильтонианов). С математической точки зрения фазовое пространство $M$ есть симплектическое многообразие размерности $2 n$, наделенное симплектической 2 -формой $\omega$. Локально оно эквивалентно симплектическому пространству $M_{0}:=\left(\mathbb{R}^{2 n}, \omega_{0}\right)$, где $\omega_{0}$ - стандартная симплектическая форма на $\mathbb{R}^{2 n}$, задаваемая в канонических координатах $\left(p_{i}, q_{i}\right), i=1, \ldots, n$, на $\mathbb{R}^{2 n}$ выражением

$$
\omega_{0}=\sum_{i=1}^{n} d p_{i} \wedge d q_{i} .
$$

Хорошо известными примерами фазовых пространств являются кокасательные расслоения и коприсоединенные орбиты групп Ли. 
Алгебра наблюдаемых $\mathcal{A}$ с математической точки зрения является произвольной подалгеброй Ли в алгебре Пуассона $C^{\infty}(M, \mathbb{R})$ гладких вещественнозначных функций на фазовом пространстве $M$ со скобкой Пуассона, определяемой симплектической 2-формой $\omega$. В частности, можно взять в качестве $\mathcal{A}$ всю алгебру Пуассона $C^{\infty}(M, \mathbb{R})$.

В случае стандартной модели $M_{0}=\left(\mathbb{R}^{2 n}, \omega_{0}\right)$ важным примером алгебры наблюдаемых $\mathcal{A}$ является алгебра Гейзенберга heis $\left(\mathbb{R}^{2 n}\right)$. Это алгебра Ли, порождаемая единицей и координатными функциями $p_{i}, q_{i}, i=1, \ldots, n$, удовлетворяющими коммутационным соотношениям

$$
\left\{p_{i}, p_{j}\right\}=\left\{q_{i}, q_{j}\right\}=0, \quad\left\{p_{i}, q_{j}\right\}=\delta_{i j}, \quad i, j=1, \ldots, n
$$

Приведем еще один пример. Предположим, что Г есть группа Ли, состоящая из симплектоморфизмов, действующих на фазовом пространстве $M$, так что алгебру Ли Lie $(\Gamma)$ можно рассматривать как подалгебру алгебры Ли гамильтоновых векторных полей на $M$. Если $M$ односвязно, то Lie(Г) можно также рассматривать двойственным образом как подалгебру алгебры Пуассона $C^{\infty}(M, \mathbb{R})$. Указанная подалгебра совпадает с алгеброй $\operatorname{Ham}(\Gamma)$ гамильтонианов (гладких вещественных функций) на $M$, порождающих симплектоморфизмы из $Г$.

ОПРЕДЕЛЕНИЕ. Квантованием Дирака классической системы $(M, \mathcal{A})$ называется неприводимое представление

$$
r: \mathcal{A} \rightarrow \text { End }^{*} H
$$

алгебры наблюдаемых $\mathcal{A}$ в алгебре линейных самосопряженных операторов, действующих в комплексном гильбертовом пространстве $H$, называемом пространством квантования. Алгебра End* $H$ наделяется скобкой Ли, задаваемой коммутатором линейных операторов вида

$$
\frac{\hbar}{i}[A, B]=\frac{\hbar}{i}(A B-B A) .
$$

Другими словами, требуется, чтобы

$$
r(\{f, g\})=\frac{\hbar}{i}(r(f) r(g)-r(g) r(f))
$$

для любых $f, g \in \mathcal{A}$. Мы также предполагаем выполненным следующее условие нормировки:

$$
r(1)=\mathrm{id}
$$

Если представление $r$ удовлетворяет всем этим требованиям, за исключением неприводимости, оно называется предквантованием системъ $(M, \mathcal{A})$.

Обычно мы полагаем для простоты $\hbar=1$. Иногда полезно рассматривать комплексифицированную алгебру наблюдаемых $\mathcal{A}^{\mathbb{C}}$ вместо $\mathcal{A}$. Ее квантование Дирака задается неприводимым представлением

$$
r: \mathcal{A}^{\mathbb{C}} \rightarrow \text { End } H
$$


удовлетворяющим условию нормировки и правилу сопряжения: $r(\bar{f})=r(f)^{*}$ для любых $f \in \mathcal{A}$.

В случае бесконечномерных алгебр наблюдаемых более естественно иметь дело с проективными представлениями алгебры наблюдаемых $\mathcal{A}$. Имея такое представление алгебры $\mathcal{A}$, мы можем построить квантование расширенной системы $(M, \tilde{\mathcal{A}})$, где $\tilde{\mathcal{A}}$ - подходящее центральное расширение алгебры $\mathcal{A}$.

Предположим, что $M$ - гладкое симплектическое многообразие размерности $2 n$ с симплектической формой $\omega, C^{\infty}(M, \mathbb{R})$ - алгебра Пуассона. Нам хотелось бы проквантовать классическую систему, задаваемую парой $\left(M, C^{\infty}(M, \mathbb{R})\right)$.

Справедливо следующее хорошо известное

ПреДЛОЖЕНИЕ. Предположим, что многообразие $M$ удовлетворяет следующему условию квантования: класс когомологий $[\omega /(2 \pi)]$ является иелочисленным в $H^{2}(M, \mathbb{R})$. Тогда существует эрмитово линейное расслоение $L \rightarrow M$, называемое расслоением предквантования, с эрмитовой связностъю $\nabla$, кривизна которой равна $\omega$.

Допустим, что наше фазовое пространство $M$ удовлетворяет условию квантования, так что выполняется утверждение предложения. Определим пространство предквантования в виде

$$
H=L^{2}\left(M, L ; \omega^{n}\right)
$$

т.е. как гильбертово пространство квадратично-интегрируемых сечений расслоения $L \rightarrow M$ со скалярным произведением, задаваемым формулой

$$
\left(s_{1}, s_{2}\right):=\int_{M}\left\langle s_{1}(x), s_{2}(x)\right\rangle \omega^{n},
$$

где $\langle\cdot, \cdot\rangle$ обозначает эрмитово произведение. Тогда KS-предквантование алгебры $\mathcal{A}=C^{\infty}(M, \mathbb{R})$ в пространстве $H$ задается формулой

$$
r_{\mathrm{KS}}: \mathcal{A} \ni f \mapsto r(f)=f-i \nabla_{X_{f}}
$$

где $X_{f}$ обозначает гамильтоново векторное поле, ассоциированное с наблюдаемой $f$. Формула (6) задает представление алгебры $\mathcal{A}=C^{\infty}(M, \mathbb{R})$ в пространстве предквантования $H$. K сожалению, это представление приводимо. "Физическое" объяснение его приводимости основано на принципе неопределенности Гейзенберга, согласно которому “физическое” пространство квантования не может содержать функций, зависящих от обеих переменных $p_{j}, q_{j}$ в какой-либо паре $\left(p_{j}, q_{j}\right)$ локальных канонических переменных (как это имеет место в пространстве предквантования $\left.H=L^{2}\left(M, L ; \omega^{n}\right)\right)$.

2.2. Действие Костанта-Сурьо на пространствах Фока. Мы можем попытаться сделать представление (6) алгебры $\mathcal{A}=C^{\infty}(M, \mathbb{R})$ в пространстве предквантования $H$ неприводимым, сужая его на "половину" пространства $H$, задаваемую каким-либо подпространством $H$, содержащим функции, зависящие только 
от одной переменной из каждой пары $\left(p_{i}, q_{i}\right)$ локальных канонических координат $\left(p_{1}, q_{1}, \ldots, p_{n}, q_{n}\right)$.

Если фазовое пространство $(M, \omega)$ является кэлеровым, т.е. обладает комплексной структурой $J$, совместимой с $\omega$, то имеется естественный выбор для указанной "половины" $H$. А именно, расслоение предквантования $L$ в этом случае можно наделить голоморфной структурой, определяемой $\bar{\partial}$-оператором, задаваемым $(0,1)$-компонентой $\nabla^{0,1}$ связности $\nabla$. Возьмем теперь в качестве “половины" $H$ подпространство $L_{\mathcal{O}}^{2}\left(M, L ; \omega^{n}\right)$ голоморфных квадратично-интегрируемых сечений расслоения $L \rightarrow M$. Тогда мы можем надеяться получить неприводимое представление алгебры наблюдаемых $\mathcal{A}$, сужая отображение $\mathrm{KS}$-предквантования на подпространство $L_{\mathcal{O}}^{2}\left(M, L ; \omega^{n}\right)$. K сожалению, этот простой подход оказывается эффективным только для весьма специальных систем, так как в большинстве случаев подпространство $L_{\mathcal{O}}^{2}\left(M, L ; \omega^{n}\right)$ не будет инвариантно относительно действия операторов представления Костанта-Сурьо (KS-операторов). Примером специальной системы, для которой указанный подход приводит к успеху, может служить стандартная модель $\mathbb{R}^{2 n} \cong \mathbb{C}^{n}$, наделенная гейзенберговой алгеброй наблюдаемых heis $\left(\mathbb{R}^{2 n}\right)=$ : heis $\left(\mathbb{C}^{n}\right)$. Сужение представления Костанта-Сурьо на подпространство $L_{\mathcal{O}}^{2}\left(\mathbb{C}^{n} ; \omega^{n}\right)$ голоморфных квадратично-интегрируемых сечений приводит в этом случае к хорошо известному неприводимому представлению Баргмана-Фока алгебры Гейзенберга heis $\left(\mathbb{C}^{n}\right)$ в пространстве $L_{\mathcal{O}}^{2}\left(\mathbb{C}^{n} ; \omega^{n}\right)$.

Однако описанный метод неприменим для общих алгебр наблюдаемых, поскольку операторы KS-предквантования в общем случае не будут сохранять фоковские пространства голоморфных сечений. Продемонстрируем это на примере кэлерова фазового пространства $(M, \omega)$, наделенного совместимой комплексной структурой $J$. Предположим, что $(M, \omega)$ удовлетворяет условию квантования и $L \rightarrow M$ - расслоение предквантования с эрмитовой связностью $\nabla$. Введем на $L$ определенную выше голоморфную структуру, задаваемую $(0,1)$-компонентой связности $\nabla$. По определению фоковское пространство

$$
F(M, J):=L_{\mathcal{O}}^{2}\left(M, L ; \omega^{n}\right)
$$

состоит из квадратично-интегрируемых сечений расслоения $L \rightarrow M$, голоморфных относительно указанной голоморфной структуры. Обозначим через $\mathcal{A}$ алгебру Ли гамильтонианов на $M$, которую можно отождествить (при условии, что $M$ односвязно) с алгеброй Ли гамильтоновых векторных полей на $M$. Любая наблюдаемая $f \in \mathcal{A}$ порождает (локальную) однопараметрическую группу Г симплектоморфизмов $M$, определяемую следующим образом:

$$
\varphi_{f}^{t}:=e^{2 \pi i t X_{f}},
$$

где $X_{f}$ - гамильтоново векторное поле, соответствующее наблюдаемой $f$. Действие группы Г на $M$ может быть поднято до действия ее центрального расширения $\widetilde{\Gamma}$ на $L$, порождаемого $\mathrm{KS}$-оператором $r(f) \equiv r_{\mathrm{KS}}(f)$. Точнее, поднятое действие задается в виде

$$
\Phi_{f}^{t}:=e^{2 \pi i t r(f)}: L^{2}\left(M, L ; \omega^{n}\right) \rightarrow L^{2}\left(M, L ; \omega^{n}\right) .
$$


Однако эти операторы не сохраняют, вообе говоря, фоковское пространство $F(M, J)$, поскольку $\Phi_{f}^{t}$ отображает фоковское пространство $F(M, J)$ в фоковское пространство $F\left(M, J_{f}^{t}\right)$, ассоциированное с преобразованной комплексной структурой $J_{f}^{t}:=\varphi_{f, \star}^{t} \circ J \circ \varphi_{f, \star}^{-t}$, которая в общем случае не эквивалентна $J$. Если структура $J_{f}^{t}$ не эквивалентна структуре $J$, то соответствующий $K S$-оператор $r_{\mathrm{KS}}(f)$ не допускает сужения на $F(M, J)$. Значит, если мы хотим построить квантование $(M, \mathcal{A})$ с помощью KS-представления, то необходимо найти метод канонического отождествления фоковских пространств $F(M, J)$ с различными $J$.

На этом строится основная идея твисторного квантования, представленная более подробно в разделе 3. Вместо того чтобы выбирать конкретную комплексную структуру на фазовом пространстве $M$, мы рассматриваем твисторное пространство всех допустимых комплексных структур на $M$. Исходная задача квантования допускает в твисторных терминах следующую переформулировку. С каждой допустимой комплексной структурой $J$ на $M$ связывается фоковское пространство $F(M, J)$, так что возникает фоковское расслоение $\mathcal{F}$ над пространством допустимых комплексных структур со слоем $F(M, J)$ в точке $J$. Предположим, что нам удалось построить плоскую эрмитову связность на этом расслоении. Тогда, пользуясь этой связностью, мы можем каноническим образом отождествить слои расслоения $\mathcal{F}$, т.е. фоковские пространства $F(M, J)$ с различными $J$.

\section{3. ПРЕДСТАВЛЕНИЯ АЛГЕБР ПЕТЕЛЬ}

3.1. Аффинные алгебры. Действие окружности $S^{1}$ играет важную роль в теории представлений алгебр петель. Для его учета удобно увеличить алгебру петель $L \mathfrak{g}$ до расширенной алгебры петель $\mathbb{C} e_{0} \oplus L \mathfrak{g}$, где $e_{0}$ обозначает генератор вращения петель. Аналогичным образом мы увеличиваем группу петель $L G$ до расширенной группы петель $U(1) \ltimes L G$, задаваемой полупрямым произведением $L G$ с группой окружности $S^{1} \equiv U(1)$.

Предположим, что $\mathfrak{g}_{\mathbb{C}}$ есть комплексная простая алгебра Ли, и зафиксируем картановскую подалгебру $\mathfrak{h}_{\mathbb{C}}$ в $\mathfrak{g}_{\mathbb{C}}$. Корневое разложение расширенной алгебры Ли $\mathbb{C} e_{0} \oplus L \mathfrak{g}_{\mathbb{C}}$ относительно картановской подалгебры $\mathbb{C} e_{0} \oplus \mathfrak{h}_{\mathbb{C}}$ имеет вид

$$
\mathbb{C} e_{0} \oplus L \mathfrak{g}_{\mathbb{C}}=\mathbb{C} e_{0} \oplus\left[\bigoplus_{n \in \mathbb{Z}} \mathfrak{h}_{\mathbb{C}} z^{n}\right] \oplus\left[\bigoplus_{(n, \alpha)} \mathfrak{g}_{\alpha} z^{n}\right]
$$

где $\mathfrak{g}_{\alpha}-$ корневые подпространства алгебры Ли $\mathfrak{g}_{\mathbb{C}}$. Пары $a=(n, \alpha)$, в которых $n \in \mathbb{Z}$, а $\alpha-$ корень $\mathfrak{g}_{\mathbb{C}}$ относительно $\mathfrak{h}_{\mathbb{C}}$, называются корнями алгебры $L \mathfrak{g}_{\mathbb{C}}$. Их можно рассматривать как линейные функционалы на алгебре Ли $\mathbb{C} e_{0} \oplus \mathfrak{h}_{\mathbb{C}}$. Если, в частности, ввести функционал $\delta \in\left(\mathbb{C} e_{0} \oplus \mathfrak{h}_{\mathbb{C}}\right)^{*}$, полагая

$$
\delta\left(e_{0}\right)=1, \quad \delta\left(\mathfrak{h}_{\mathbb{C}}\right)=0,
$$

то все множество корней алгебры $\mathbb{C} e_{0} \oplus L \mathfrak{g}_{\mathbb{C}}$ относительно $\mathbb{C} e_{0} \oplus \mathfrak{h}_{\mathbb{C}}$ будет совпадать с

$$
\hat{\Delta}=\{\alpha+n \delta: \alpha \in \Delta, n \in \mathbb{Z}\} \cup\{n \delta: n \in \mathbb{Z}\},
$$


где $\Delta-$ множество корней алгебры $\mathfrak{g}_{\mathbb{C}}$ относительно $\mathfrak{h}_{\mathbb{C}}$. Соответственно множество положительных корней алгебры $\mathbb{C} e_{0} \oplus L \mathfrak{g}_{\mathbb{C}}$ относительно $\mathbb{C} e_{0} \oplus \mathfrak{h}_{\mathbb{C}}$ отождествляется с

$$
\hat{\Delta}^{+}=\{\alpha+n \delta: \alpha \in \Delta, n>0\} \cup\{n \delta: n>0\} \cup \Delta^{+},
$$

где $\Delta^{+}-$множество положительных корней алгебры $\mathfrak{g}_{\mathbb{C}}$ относительно $\mathfrak{h}_{\mathbb{C}}$. Если $\left\{\alpha_{1}, \ldots, \alpha_{l}\right\}$ есть система простых корней алгебры $\mathfrak{g}_{\mathbb{C}}$ относительно $\mathfrak{h}_{\mathbb{C}}, A$ - старший вес в $\Delta^{+}$, то любой корень в $\hat{\Delta}^{+}$будет записываться в виде

$$
n_{0} \alpha_{0}+n_{1} \alpha_{1}+\cdots+n_{l} \alpha_{l}
$$

с целыми неотрицательными коэффициентами $n_{0}, n_{1}, \ldots, n_{l}$, где $\alpha_{0}:=\delta-A$. Будем называть $\left\{\alpha_{0}, \alpha_{1}, \ldots, \alpha_{l}\right\}$ системой аффинных простых корней в $\hat{\Delta}$.

Сопоставим произвольному корню $a=(n, \alpha)$ корневое подпространство $\mathfrak{g}_{(n, \alpha)}$ в $L \mathfrak{g}_{\mathbb{C}}$, задаваемое в виде

$$
\begin{array}{rlrl}
\mathfrak{g}_{(n, \alpha)} & =\mathfrak{g}_{\alpha} z^{n} & \text { при } & \alpha \neq 0, \\
\mathfrak{g}_{(n, 0)}=\mathfrak{h}_{\mathbb{C}} z^{n} & \text { при } & \alpha=0 .
\end{array}
$$

Петлевой аналог разложения алгебры Ли $\mathfrak{g}_{\mathbb{C}}$,

$$
\mathfrak{g}_{\mathbb{C}}=\mathfrak{h}_{\mathbb{C}} \oplus \mathfrak{n}^{+} \oplus \mathfrak{n}^{-},
$$

где $\mathfrak{n}^{ \pm}$- нильпотентные подалгебры алгебры Ли $\mathfrak{g}_{\mathbb{C}}$ вида

$$
\mathfrak{n}^{+}=\bigoplus_{\alpha \in \Delta^{+}} \mathfrak{g}_{\alpha}, \quad \mathfrak{n}^{-}=\bigoplus_{\alpha \in \Delta^{-}} \mathfrak{g}_{\alpha}
$$

имеет вид

$$
L \mathfrak{g}_{\mathbb{C}}=\mathfrak{h}_{\mathbb{C}} \oplus N^{+} \mathfrak{g}_{\mathbb{C}} \oplus N^{-} \mathfrak{g}_{\mathbb{C}}
$$

где

$$
N^{+} \mathfrak{g}_{\mathbb{C}}=\mathfrak{n}^{+} \oplus\left[\bigoplus_{n>0} \mathfrak{g}_{\mathbb{C}} \cdot z^{n}\right], \quad N^{-} \mathfrak{g}_{\mathbb{C}}=\mathfrak{n}^{-} \oplus\left[\bigoplus_{n<0} \mathfrak{g}_{\mathbb{C}} \cdot z^{n}\right] .
$$

Петлевые аналоги борелевских подалгебр задаются как

$$
B^{ \pm} \mathfrak{g}_{\mathbb{C}}=\mathfrak{h}_{\mathbb{C}} \oplus N^{ \pm} \mathfrak{g}_{\mathbb{C}}
$$

Центральное расширение $\widetilde{L \mathfrak{g}_{\mathbb{C}}}$ алгебры петель $L \mathfrak{g}_{\mathbb{C}}$ определяется 2-коциклом на алгебре Ли $L \mathfrak{g}_{\mathbb{C}}$, задаваемым формулой

$$
\omega(\xi, \eta)=\omega_{0}(\xi, \eta)=\frac{1}{2 \pi} \int_{0}^{2 \pi}\left\langle\xi\left(e^{i \theta}\right), \eta^{\prime}\left(e^{i \theta}\right)\right\rangle d \theta, \quad \xi, \eta \in L \mathfrak{g}_{\mathbb{C}}
$$

где $\langle\cdot, \cdot\rangle$ - инвариантное скалярное произведение на алгебре Ли $\mathfrak{g}_{\mathbb{C}}$. Как векторное пространство,

$$
\widetilde{L \mathfrak{g}_{\mathbb{C}}}=L \mathfrak{g}_{\mathbb{C}} \oplus \mathbb{C} c
$$


с коммутационными соотношениями

$$
[\xi+s c, \eta+t c]=[\xi, \eta]+\omega(\xi, \eta) c
$$

для $\xi, \eta \in L \mathfrak{g}_{\mathbb{C}}, s, t \in \mathbb{C}$. Пусть $\widetilde{L G_{\mathbb{C}}}$ - центральное расширение группы петель $L G_{\mathbb{C}}$, соответствующее указанному центральному расширению алгебры петель $L \mathfrak{g}_{\mathbb{C}}$.

Рассматриваемые здесь представления алгебры петель $L \mathfrak{g}$ и группы петель $L G$ являются проективными и согласованы с $S^{1}$-действием. Это означает, что они возникают в действительности из представлений аффинной алгебры

$$
\widehat{L \mathfrak{g}_{\mathbb{C}}}=\mathbb{C} e_{0} \oplus \widetilde{L \mathfrak{g}_{\mathbb{C}}}=\mathbb{C} e_{0} \oplus L \mathfrak{g}_{\mathbb{C}} \oplus \mathbb{C} c
$$

и аффинной группы

$$
\widehat{L G_{\mathbb{C}}}:=\mathbb{C}^{*} \ltimes \widetilde{L G_{\mathbb{C}}}
$$

Корневое разложение аффинной алгебры $\widehat{\lg _{\mathbb{C}}}$ имеет вид

$$
\widehat{L \mathfrak{g}_{\mathbb{C}}}=\widehat{\mathfrak{h}_{\mathbb{C}}} \oplus N^{+} \mathfrak{g}_{\mathbb{C}} \oplus N^{-} \mathfrak{g}_{\mathbb{C}}
$$

где

$$
\widehat{\mathfrak{h}_{\mathbb{C}}}=\mathbb{C} e_{0} \oplus \widetilde{\mathfrak{h}_{\mathbb{C}}}=\mathbb{C} e_{0} \oplus \mathfrak{h}_{\mathbb{C}} \oplus \mathbb{C} c
$$

Соответственно

$$
\widehat{B^{ \pm} \mathfrak{g}_{\mathbb{C}}}=\widehat{\mathfrak{h}_{\mathbb{C}}} \oplus N^{ \pm} \mathfrak{g}_{\mathbb{C}}
$$

Имея корень $\alpha \in \mathfrak{h}_{\mathbb{C}}^{*}$, мы можем продолжить его на $\widehat{\mathfrak{h}}_{\mathbb{C}}^{*}$, полагая $\alpha(c)=\alpha\left(e_{0}\right)=0$. Продолжим также функционал $\delta \in\left(\mathbb{C} e_{0} \oplus \mathfrak{h}_{\mathbb{C}}\right)^{*}$ на $\widehat{\mathfrak{h}}_{\mathbb{C}}^{*}$, полагая $\delta(c)=0$. Введем функционал $\beta \in \widehat{\mathfrak{h}}_{\mathbb{C}}^{*}$ :

$$
\beta(c)=1, \quad \beta\left(e_{0}\right)=0, \quad \beta\left(\mathfrak{h}_{\mathbb{C}}\right)=0 .
$$

Мы можем сопоставить любой системе $\alpha_{0}, \alpha_{1}, \ldots, \alpha_{l}$ аффинных простых корней отвечающую ей систему кокорней $\alpha_{0}^{\vee}, \alpha_{1}^{\vee}, \ldots, \alpha_{l}^{\vee}$, где $\alpha_{j}^{\vee}, j=1, \ldots, l,-$ кокорень, ассоциированный с простым корнем $\alpha_{j}$ алгебры $\mathfrak{g}_{\mathbb{C}}$, a

$$
\alpha_{0}^{\vee}=-A^{\vee}+\frac{2 c}{\langle A, A\rangle}
$$

- аффинный кокорень, ассоциированный со старшим корнем $A \in \Delta^{+}$.

Обозначим через $\left\{\omega_{1}, \ldots, \omega_{l}\right\}$ систему фундаментальных весов алгебры $\mathfrak{g}_{\mathbb{C}}$, двойственную к системе простых корней $\alpha_{1}, \ldots, \alpha_{l}$. Ассоциированная система $\left\{\widehat{\omega}_{0}, \widehat{\omega}_{1}, \ldots, \widehat{\omega}_{l}\right\}$ фундаментальных весов аффинной алгебры $\widehat{L \mathfrak{g}_{\mathbb{C}}}$, двойственная к системе $\alpha_{0}, \alpha_{1}, \ldots, \alpha_{l}$ аффинных простых корней, определяется следующим образом:

$$
\widehat{\omega}_{i}\left(\alpha_{k}^{\vee}\right)=\delta_{i k} \quad \text { для } \quad 0 \leqslant i, k \leqslant l, \quad \widehat{\omega}_{i}\left(e_{0}\right)=0 .
$$

Тогда

$$
\widehat{\omega}_{0}=\frac{1}{2}\langle A, A\rangle \beta, \quad \widehat{\omega}_{j}=\omega_{j}+\left\langle\omega_{j}, A\right\rangle \beta, \quad 1 \leqslant j \leqslant l .
$$


3.2. Представления старшего веса аффинных алгебр. Предположим, что $\rho: \widehat{L \mathfrak{g}_{\mathbb{C}}} \rightarrow V$ есть представление алгебры петель $\widehat{L \mathfrak{g}_{\mathbb{C}}}$, т.е. $\widehat{L \mathfrak{g}_{\mathbb{C}}}$-модуль. Рассмотрим для любой линейной формы на $\widehat{\mathfrak{h}}_{\mathbb{C}}$, т.е. элемента $\lambda \in \widehat{\mathfrak{h}}_{\mathbb{C}}^{*}$, подпространство

$$
V_{\lambda}=\left\{v \in V: \rho(h) v=\lambda(h) v \quad \text { для } \quad h \in \widehat{\mathfrak{h}}_{\mathbb{C}}\right\} .
$$

Если $V_{\lambda} \neq 0$, то $\lambda$ называется весом $\rho$, а подпространство $V_{\lambda}$ - весовым подпространством $\rho$, отвечающим $\lambda$. Любой вектор $v \in V_{\lambda} \backslash\{0\}$ называется весовым вектором $\rho$.

Функционал $\lambda \in \widehat{\mathfrak{h}}_{\mathbb{C}}^{*}$ называется целочисленным доминантным весом, если $\lambda\left(\alpha_{i}^{\vee}\right)$ является целым неотрицательным числом для любого аффинного кокорня $\alpha_{i}^{\vee}, 0 \leqslant$ $i \leqslant l$. Любой такой вес может быть записан в виде

$$
\lambda=n_{0} \widehat{\omega}_{0}+\cdots+n_{l} \widehat{\omega}_{l}+s \delta
$$

где $n_{i}=\lambda\left(\alpha_{i}^{\vee}\right), 0 \leqslant i \leqslant l, s=\lambda\left(e_{0}\right) \in \mathbb{C}$. Соответственно антидоминантный целочисленный вес $\lambda \in \widehat{\mathfrak{h}}_{\mathbb{C}}^{*}$ принимает неположительные целочисленные значения на аффинных кокорнях $\alpha_{i}^{\vee}, 0 \leqslant i \leqslant l$, и может быть записан в форме (8) с неположительными целочисленными коэффициентами $n_{i}, 0 \leqslant i \leqslant l$.

Мы можем продолжить вес $\lambda \in \widehat{\mathfrak{h}}_{\mathbb{C}}^{*}$ на борелевскую подалгебру $\widehat{B^{+} \mathfrak{g}_{\mathbb{C}}}$, полагая его равным нулю на $N^{+} \mathfrak{g}_{\mathbb{C}}$. Рассмотрим $\widehat{L \mathfrak{g}_{\mathbb{C}}}$-модуль вида

$$
\widehat{V} \equiv \widehat{V}_{\lambda}=\mathcal{U}\left(\widehat{L \mathfrak{g}_{\mathbb{C}}}\right) \bigotimes_{\mathcal{U}\left(\widehat{B^{+} \mathfrak{g}_{\mathbb{C}}}\right)} \mathbb{C}_{\lambda}
$$

где символ $\mathcal{U}$ обозначает универсальную обертывающую алгебру, $\mathbb{C}_{\lambda}$ - одномерный $\widehat{B^{+} \mathfrak{g}_{\mathbb{C}}}$-модуль, т.е. комплексную прямую $\mathbb{C}$, наделенную действием борелевской подалгебры $\widehat{B^{+} \mathfrak{g}_{\mathbb{C}}}$, задаваемым соотношением $z \mapsto \lambda(b) z$ для $b \in \widehat{B^{+} \mathfrak{g}^{\mathbb{C}}}, z \in \mathbb{C}$. Поскольку

$$
\widehat{L \mathfrak{g}_{\mathbb{C}}}=N^{-} \mathfrak{g}_{\mathbb{C}} \oplus \widehat{B^{+} \mathfrak{g}_{\mathbb{C}}}
$$

из теоремы Пуанкаре-Биркгофа-Витта вытекает, что

$$
\mathcal{U}\left(\widehat{L \mathfrak{g}_{\mathbb{C}}}\right) \cong \mathcal{U}\left(N^{-} \mathfrak{g}_{\mathbb{C}}\right) \otimes \mathcal{U}\left(\widehat{B^{+} \mathfrak{g}_{\mathbb{C}}}\right)
$$

Тем самым имеется естественный изоморфизм

$$
\widehat{V}_{\lambda} \cong \mathcal{U}\left(N^{-} \mathfrak{g}_{\mathbb{C}}\right) \otimes \mathbb{C}_{\lambda}
$$

Обозначим через $V \equiv V_{\lambda}$ фактор $\widehat{V}$ по максимальному подмодулю в $\widehat{V}$, строго содержащемуся в $\widehat{V}$ (иными словами, по максимальному подмодулю, не содержащему $1 \otimes 1)$. Это пространство $V$, наделенное естественным действием алгебры $\widehat{L \mathfrak{g}_{\mathbb{C}}}$, называется стандартным представлением алгебры Ли $\widehat{L \mathfrak{g}_{\mathbb{C}}}$ со старшим весом $\lambda$ и весовым вектором $1 \otimes 1$.

Будем говорить, что представление $\rho: \widehat{L \mathfrak{g}_{\mathbb{C}}} \rightarrow$ End $V_{\lambda}$ аффинной алгебры $\widehat{L \mathfrak{g}_{\mathbb{C}}}$ является представлением старшего веса $\lambda \in \widehat{\mathfrak{h}}_{\mathbb{C}}^{*}$, если существует вектор старшего веса $v_{\lambda} \in V_{\lambda}$ такой, что:

1) $\rho(h) v_{\lambda}=\lambda(h) v_{\lambda}$ для любого $h \in \widehat{\mathfrak{h}_{\mathbb{C}}}$; 
2) $\rho(n) v_{\lambda}=0$ для любого $n \in N^{+} \mathfrak{g}_{\mathbb{C}}$;

3) $V_{\lambda}$ порождается векторами вида $\rho(b) v_{\lambda}$ с $b \in \widehat{B^{-} \mathfrak{g}_{\mathbb{C}}}$.

Вектор старшего веса $v_{\lambda}$ играет роль, аналогичную вакууму для представления Гейзенберга.

Подобным же образом можно определить представления младшего веса аффинной алгебры $\widehat{L \mathfrak{g}_{\mathbb{C}}}$. Для этого нужно заменить в приведенном выше определении нильпотентную подалгебру $N^{+} \mathfrak{g}_{\mathbb{C}}$ на нильпотентную подалгебру $N^{-} \mathfrak{g}_{\mathbb{C}}$ и борелевскую подалгебру $\widehat{B^{-} \mathfrak{g}_{\mathbb{C}}}$ на борелевскую подалгебру $\widehat{B^{+} \mathfrak{g}_{\mathbb{C}}}$.

Определенный выше стандартный $\widehat{L \mathfrak{g}_{\mathbb{C}}}$-модуль $V_{\lambda}$ является неприводимым представлением старшего веса алгебры $\widehat{L \mathfrak{g}_{\mathbb{C}}}$, если вес $\lambda \in \widehat{\mathfrak{h}}_{\mathbb{C}}^{*}$ целочисленный доминантный. Более того, если значение $\lambda\left(e_{0}\right)$ вещественно, то $V_{\lambda}$ допускает положительно определенное (контравариантное) эрмитово скалярное произведение. Обозначим через $H \equiv H_{\lambda}$ пополнение $V \equiv V_{\lambda}$ относительно этого скалярного произведения. Пространство $H_{\lambda}$ будет играть роль фоковского пространства, ассоциированного с весом $\lambda$.

\section{4. ПРЕДСТАВЛЕНИЯ ГРУПП ПЕТЕЛЬ}

4.1. Неприводимые представления аффинных групп. Рассмотрим аффинную группу

$$
\widehat{L G}:=U(1) \ltimes \widetilde{L G}
$$

и зафиксируем максимальный тор $\widehat{T}$ в $\widehat{L G}$ вида

$$
\widehat{T}:=U(1) \times T \times S .
$$

Здесь $U(1)=S^{1}$ соответствует группе поворотов, $T$ есть максимальный тор в группе $G$, а $S=S^{1}$ задает центральную подгруппу в группе $\widetilde{L G}$.

Любое неприводимое представление аффинной группы $\widehat{L G}$ обладает единственным старшим весом $\lambda$, являющимся характером максимального тора $\widehat{T}$. Указанный характер имеет вид

$$
\lambda=\left(n, \lambda_{0}, h\right),
$$

где $n \in \mathbb{Z}$ есть собственное значение оператора поворота $e_{0}$, называемое энергией представления, $\lambda_{0}$ - характер $T, h \in \mathbb{Z}$ - собственное значение относительно действия центральной подгруппы, называемое уровнем представления. Старшие веса $\widehat{L G}$ являются целочисленными доминантными, классы изоморфизма неприводимых представлений группы $\widehat{L G}$ находятся во взаимнооднозначном соответствии с множеством целочисленных доминантных весов.

Имеется аналогичное описание неприводимых представлений аффинной группы $\widehat{L G}$ в терминах младших весов.

4.2. Конструкция Бореля-Вейля. Рассмотрим пространство петель

$$
\Omega_{T} G=L G / T=L G^{\mathbb{C}} / B^{+} G^{\mathbb{C}},
$$


или, в терминах центральных расширений,

$$
\Omega_{T} G=\widetilde{L G^{\mathbb{C}}} / \widetilde{B^{+} G^{\mathbb{C}}} .
$$

Предположим, что $\lambda$ - младший вес максимального тора $\widetilde{T}=T \times S$. Продолжим его на $\widehat{B^{+} G^{\mathbb{C}}}$, полагая $\lambda=1$ на нильпотентной подгруппе $N^{+} G^{\mathbb{C}}$ в

$$
\widetilde{B^{+} G^{\mathbb{C}}}=\widetilde{T^{\mathbb{C}}} \times N^{+} G^{\mathbb{C}} \text {. }
$$

Определим голоморфное линейное расслоение $L=L_{\lambda}$ над $\Omega_{T} G$ в виде

$$
L=\widetilde{L G^{\mathbb{C}}} \times \widetilde{B^{+} G^{\mathbb{C}}} \mathbb{C} \rightarrow \Omega_{T} G=\widetilde{L G^{\mathbb{C}}} / \widetilde{B^{+} G^{\mathbb{C}}},
$$

где $\widetilde{B^{+} G^{\mathbb{C}}}$ действует на комплексной прямой $\mathbb{C}$ с помощью характера $\lambda$ :

$$
\widetilde{B^{+} G^{\mathbb{C}}} \ni b \mapsto \lambda(b) z
$$

Обозначим через $\Gamma=\Gamma_{\lambda}$ векторное пространство голоморфных сечений расслоения $L=L_{\lambda}$. Сечения $s \in \Gamma$ можно отождествить с голоморфными функциями $\dot{s}: \widetilde{L G^{\mathbb{C}}} \rightarrow \mathbb{C}$, удовлетворяющими условию

$$
\dot{s}\left(\gamma b^{-1}\right)=\lambda(b) \dot{s}(\gamma)
$$

для любых $b \in \widetilde{B^{+} G^{\mathbb{C}}}, \gamma \in \widetilde{L G^{\mathbb{C}}}$. Группа $\widetilde{L G^{\mathbb{C}}}$ действует естественным образом на расслоении $L$ и на пространстве $\Gamma$, и это действие задает голоморфное представление группы $\widetilde{L G^{\mathbb{C}}}$ на Г. Более того, пространство Г является нетривиальным (т.е. содержит голоморфные сечения $L$, не равные тождественно нулю) тогда и только тогда, когда вес $\lambda$ является целочисленным антидоминантным [2]. При этом условии можно показать, что соответствующее представление группы петель $\widetilde{L G}$ является неприводимым представлением $\widetilde{L G}$ младшего веса $\lambda[2]$.

Заметим, что $\Gamma$ содержит одномерное подпространство сечений, инвариантных относительно действия нильпотентной подгруппы $N^{-} G^{\mathbb{C}}$. Действительно, из представления (4) вытекает, что в $\Omega_{T} G$ имеется открытая плотная орбита, содержащая начало $o \in \Omega_{T} G$, которую можно отождествить с подгруппой $N^{-} G^{\mathbb{C}}$. Следовательно, любое $N^{-} G^{\mathbb{C}}$-инвариантное сечение из $\Gamma$ однозначно определяется своим значением в начале. Выберем в качестве вакуума вектор младшего веса $v=v_{\lambda}$, который совпадает с $N^{-} G^{\mathbb{C}}$-инвариантным сечением в $\Gamma$, равным единице в начале $o$.

На $Г$ имеется эрмитово скалярное произведение, определенное на плотном подмножестве. Действительно, рассмотрим антидвойственное пространство $\bar{\Gamma}^{*}$ и определим комплексно-линейное отображение $\beta: \bar{\Gamma}^{*} \rightarrow \Gamma$, значение которого на элементе $\xi \in \bar{\Gamma}^{*}$ является сечением $\beta(\xi) \in \Gamma$, отождествляемым с функцией $\dot{\beta}(\xi)$ на $\widetilde{L G^{\mathbb{C}}}$, задаваемой равенством

$$
\dot{\beta}(\xi)(\gamma):=\xi(\gamma \cdot v) \quad \text { для } \quad \gamma \in \widetilde{L G^{\mathbb{C}}} \text {. }
$$


С помощью этого отображения определим эрмитово скалярное произведение элементов $\xi, \eta \in \bar{\Gamma}^{*}$, полагая

$$
\langle\xi, \eta\rangle:=\eta(\overline{\beta(\xi)}) .
$$

Построенное скалярное произведение на $\bar{\Gamma}^{*}$ положительно определено. Обозначим через $H=H_{\lambda}$ замыкание $\bar{\Gamma}^{*}$ относительно этого скалярного произведения, так что $\bar{\Gamma}^{*} \subset H \subset \Gamma$. Пространство $H$ играет роль фоковского пространства, ассоциированного с младшим весом $\lambda$.

\section{5. ТВИСТОРНОЕ КВАНТОВАНИЕ ПРОСТРАНСТВА $\Omega_{T} G$}

Наша цель - проквантовать классическую систему $\left(\Omega_{T} G, \widehat{L \mathfrak{g}} \rtimes\right.$ vir $)$ с фазовым пространством $\Omega_{T} G$ и алгеброй наблюдаемых $\mathcal{A}$, задаваемой полупрямым произведением аффинной алгебры $\widehat{L \mathfrak{g}}$ и алгебры Вирасоро vir, являющейся центральным расширением алгебры $\operatorname{Vect}\left(S^{1}\right)$ касательных векторных полей на $S^{1}$. Воспользуемся для этого конструкцией Гудмана-Уолаха, дающей проективное действие группы диффеоморфизмов $\operatorname{Diff}_{+}\left(S^{1}\right)$ на представлениях аффинной алгебры $\widehat{L \mathfrak{g}^{\mathbb{C}}}$ и аффинной группы $\widehat{L G^{\mathbb{C}}}$.

5.1. Конструкция Гудмана-Уолаха. Продолжим естественное правое действие группы Diff $+\left(S^{1}\right)$ на $L \mathfrak{g}^{\mathbb{C}}$ на алгебру $\widetilde{L \mathfrak{g}^{\mathbb{C}}}$ так, чтобы действие группы Diff $+\left(S^{1}\right)$ на центральной подалгебре в $\widetilde{L \mathfrak{g}^{\mathbb{C}}}$ было тривиально. Для $f \in \operatorname{Diff}_{+}\left(S^{1}\right)$ обозначим действие $f$ на $\widetilde{L \mathfrak{g}^{\mathbb{C}}}$ через $\xi \mapsto \xi_{f}$ при $\xi \in \widetilde{L \mathfrak{g}^{\mathbb{C}}}$.

Для заданного представления младшего веса $(V, \pi) \equiv\left(V_{\lambda}, \pi_{\lambda}\right)$ алгебры $\widetilde{L \mathfrak{g}^{\mathbb{C}}}$ определим действие $f \in \operatorname{Diff}_{+}\left(S^{1}\right)$ на $(V, \pi)$, полагая

$$
f: \pi \mapsto \pi_{f},
$$

где $\pi_{f}(\xi) v:=\pi\left(\xi_{f}\right) v$ при $\xi \in \widetilde{L \mathfrak{g}^{\mathbb{C}}}, v \in V$. Основной результат работы ГудманаУолаха [1] утверждает, что представления $\pi$ и $\pi_{f}$ унитарно эквивалентны. Точнее, существует унитарное проективное действие $\sigma$ группы $\operatorname{Diff}_{+}\left(S^{1}\right)$ на $H \equiv H_{\lambda}$ такое, что на плотном подпространстве $H^{\infty} \subset H$ выполняется равенство $\sigma(f) \pi_{f}(\xi) v=$ $\pi(\xi) \sigma(f) v$ для любых $v \in H^{\infty}, f \in \operatorname{Diff}_{+}\left(S^{1}\right), \xi \in \widetilde{L \mathfrak{g}^{\mathbb{C}}}$.

5.2. Твисторное квантование пространства $\Omega_{T} G$. В п. 4.2 мы построили для любого младшего веса $\lambda$ алгебры петель $\widetilde{L g}$ голоморфное линейное расслоение $L \equiv L_{\lambda} \rightarrow \Omega_{T} G$ и пространство $\Gamma \equiv \Gamma_{\lambda}$ его голоморфных сечений, на котором реализуется представление группы $\widetilde{L G}$ младшего веса $\lambda$, и обозначили через $H \equiv H_{\lambda}$ пополнение $\bar{\Gamma}^{*}$ по естественной норме.

Указанная конструкция зависит от комплексной структуры на пространстве $\Omega_{T} G$, задаваемой комплексным представлением

$$
\Omega_{T} G=L G^{\mathbb{C}} / B^{+} G^{\mathbb{C}} .
$$

Обозначим эту комплексную структуру через $J_{0}$, а отвечающие ей пространства $\Gamma_{\lambda}$ и $H_{\lambda}$ через $\Gamma_{0}$ и $H_{0}$ соответственно, так что у нас имеется представление $\pi_{0}$ группы $\widetilde{L G}$ в пространстве $\Gamma_{0}$. 
Изменяя эту комплексную структуру на структуру $J_{f}$, полученную из $J_{0}$ действием диффеоморфизма $f \in \operatorname{Diff}_{+}\left(S^{1}\right)$, мы можем снова, пользуясь конструкцией Бореля-Вейля, реализовать представление младшего веса $\pi_{f}$ группы $\widetilde{L G}$, отвечающее младшему весу $\lambda$, в пространстве $\Gamma_{f}$ сечений расслоения $L$, голоморфных относительно комплексной структуры $J_{f}$ на $\Omega_{T} G$. Обозначим соответствующее пополнение пространства $\bar{\Gamma}_{f}^{*}$ через $H_{f}$.

Согласно конструкции Гудмана-Уолаха имеется проективное унитарное действие

$$
U_{f}: \Gamma_{0} \rightarrow \Gamma_{f}
$$

группы Diff $+\left(S^{1}\right)$, сплетающее представления $\pi_{0}$ и $\pi_{f}$. Оно однозначно определяется условием нормировки, налагаемым на векторы младшего веса: $U_{f} v_{0}=v_{f}$.

Тем самым имеется голоморфное гильбертово расслоение

$$
H=\bigcup_{f \in \mathcal{S}} H_{f}
$$

и проективное унитарное действие группы $\operatorname{Diff}_{+}\left(S^{1}\right)$ на $H$, задаваемое отображением $f \mapsto U_{f}$, которое накрывает естественное $\operatorname{Diff}_{+}\left(S^{1}\right)$-действие на $\mathcal{S}$. Инфинитезимальная версия этого действия дает проективное унитарное представление $\rho$ младшего веса $\lambda$ алгебры Ли $\operatorname{Vect}\left(S^{1}\right)$ в пространстве $H_{0}$ (явное описание этого представления дано в [1]).

Имея проективное представление $\rho$ алгебры $\operatorname{Vect}\left(S^{1}\right)$, мы можем построить $\operatorname{Diff}_{+}\left(S^{1}\right)$-инвариантную связность А на расслоении $H \rightarrow \mathcal{S}$, кривизна которой в начале $o \in \mathcal{S}$ совпадает с коциклом представления $\rho$. Указанный коцикл был вычислен в работах [5], [6]. Он задается в базисе $\left\{e_{n}:=i e^{i n \theta} d / d \theta\right\}$ комплексифицированной алгебры $\operatorname{Vect}^{\mathbb{C}}\left(S^{1}\right)$ формулой

$$
\left[\rho\left(e_{m}\right), \rho\left(e_{n}\right)\right]-\rho\left(\left[e_{m}, e_{n}\right]\right)=\frac{c(\mathfrak{g})}{12}\left(m^{3}-m\right) \delta_{m,-n}
$$

где

$$
c(\mathfrak{g})=\frac{h \operatorname{dim} \mathfrak{g}}{h+\kappa(\mathfrak{g})},
$$

$\kappa(\mathfrak{g})$ есть двойственное число Кокстера алгебры $\mathfrak{g}$ (см., например, [7]).

Для построения связности А заметим, что имеется разложение алгебры Ли $\operatorname{Vect}\left(S^{1}\right)$ в прямую сумму

$$
\operatorname{Vect}\left(S^{1}\right)=\operatorname{sl}(2, \mathbb{R}) \oplus \mathfrak{m}
$$

где $\operatorname{sl}(2, \mathbb{R})$ есть алгебра Ли группы $\operatorname{Möb}\left(S^{1}\right)$, a $\mathfrak{m}$ отождествляется с касательным пространством $T_{o} \mathcal{S}$. Присоединенное действие группы $\operatorname{Möb}\left(S^{1}\right)$ на $\operatorname{Vect}\left(S^{1}\right)$ сохраняет подпространство $\mathfrak{m}$. Согласно общей теории инвариантных связностей ([8], гл. II.11) разложение (9) и проективное представление $\rho$ определяют $\operatorname{Diff}_{+}\left(S^{1}\right)$ инвариантную связность $\mathbf{A}$ на расслоении $H$ с кривизной, равной коциклу $\rho$. 
5.3. Расслоение квантования. Обозначим через $K \rightarrow \mathcal{S}$ каноническое расслоение многообразия $\mathcal{S}[9]$. Оно наделяется эрмитовой метрикой $\tilde{g}$, индуцированной кэлеровой метрикой $g$ на $\mathcal{S}$. Кроме того, на $K$ имеется естественное действие группы Вирасоро Vir, являющейся центральным расширением группы $\operatorname{Diff}_{+}\left(S^{1}\right)$, которое накрывает действие $\operatorname{Diff}_{+}\left(S^{1}\right)$ левыми сдвигами на $\mathcal{S}$. Каноническая связность на $K$, порождаемая метрикой $\tilde{g}$, инвариантна относительно указанного действия.

Обозначим через $K^{-1} \rightarrow \mathcal{S}$ двойственное антиканоническое расслоение на $\mathcal{S}$. Поскольку $\mathcal{S}$ стягиваемо, то антиканоническое расслоение $K^{-1}$ допускает квадратный корень $K^{-1 / 2} \rightarrow \mathcal{S}$. Метрика $\tilde{g}$ на $K$ индуцирует эрмитову метрику на $K^{-1 / 2}$. Группа Вирасоро Vir действует на $K^{-1 / 2}$, и каноническая связность В на $K^{-1 / 2} \rightarrow \mathcal{S}$, порождаемая указанной эрмитовой метрикой, инвариантна относительно этого действия. Кривизна связности $\mathbf{B}$ на $K^{-1 / 2} \rightarrow \mathcal{S}$ вычисляется в базисе $\left\{e_{n}\right\}$ по формуле $[10]$

$$
R_{\mathbf{B}}\left(e_{m}, e_{n}\right)=-\frac{26}{12}\left(m^{3}-m\right) \delta_{m,-n} .
$$

Определим расслоение квантования $\mathcal{H}$ в виде

$$
\mathcal{H}:=H \otimes K^{-1 / 2} \rightarrow \mathcal{S}
$$

и наделим его связностью тензорного произведения

$$
\mathbf{C}:=\mathbf{A} \otimes 1+1 \otimes \mathbf{B}
$$

Кривизна связности $\mathbf{C}$ в базисе $\left\{e_{n}\right\}$ равна

$$
R_{\mathbf{C}}\left(e_{m}, e_{n}\right)=\frac{c(\mathfrak{g})-26}{12}\left(m^{3}-m\right) \delta_{m,-n}
$$

и равна нулю при $c(\mathfrak{g})=26$. При этом условии мы получаем плоскую эрмитову связность на расслоении $\mathcal{H}$, дающую решение задачи твисторного квантования для нашей классической системы $\left(\Omega_{T} G, \mathcal{A}=\widehat{L \mathfrak{g}} \rtimes\right.$ vir $)$.

Как можно получить решение исходной задачи дираковского квантования для системы $\left(\Omega_{T} G, \mathcal{A}\right)$ исходя из найденного решения задачи твисторного квантования? Как мы уже отмечали ранее, KS-операторы, ассоциированные с элементами алгебры Ли vir группы Вирасоро Vir, не сохраняют, вообще говоря, пространства $H_{0}$ и $\mathcal{H}_{0}$. Точнее, симплектоморфизмы $\varphi^{t}$, порожденные этими элементами, переводят указанные пространства в пространства $H_{t}$ и $\mathcal{H}_{t}$ соответственно, отвечающие комплексной структуре $J_{t}:=\varphi_{*}^{t} \circ J_{0} \circ\left(\varphi_{*}^{t}\right)^{-1}$. Интегрируя построенную плоскую эрмитову связность на расслоении предквантования $\mathcal{H}$, можно определить унитарный оператор $U_{t}$, отождествляющий $\mathcal{H}_{t}$ с $\mathcal{H}_{0}$. Композиция $U_{t}$ с элементами алгебры vir, действующая теперь в пространстве $\mathcal{H}_{0}$, даст нам после дифференцирования требуемое представление алгебры Вирасоро vir в пространстве $\mathcal{H}_{0}$.

Благодарности. Работа выполнена при поддержке РФФИ (гранты № 06-0204012, № 08-01-00014), Программы поддержки ведущих научных школ (грант НШ3224.2008.1) и научной программы Президиума РАН "Нелинейная динамика". 


\section{Список литературы}

[1] R. Goodman, N. R. Wallach, J. Reine Angew. Math., 347 (1984), 69-133.

[2] Э. Прессли, Г. Сигал, Группь петель, Мир, М., 1990.

[3] А.А. Кириллов, Элементы теории представлений, Наука, М., 1978.

[4] А. Г. Сергеев, Кэлерова геометрия пространства петель, МНЦМО, М., 2001.

[5] J. Mickelsson, Comm. Math. Phys., 112:4 (1987), 653-661.

[6] J. Mickelsson, Current Algebras and Groups, Plenum Monogr. Nonlinear Phys., Plenum Press, New York, 1989.

[7] А.Л. Онищик, Е.Б. Винберг, Семинар по группам Ли и алгебраическим группам, Наука, М., 1988.

[8] Ш. Кобаяси, К. Номидзу, Основы дифференциалъной геометрии, Т. 1, 2, Наука, М., 1981.

[9] G. Segal, Comm. Math. Phys., 80:3 (1981), 301-342.

[10] M. J. Bowick, A. Lahiri, J. Math. Phys., 29:9 (1988), 1979-1981.

Поступила в редакцию 29.05.2008 